\title{
PART 2M
}

\section{HIV}

\section{E J Smit}

A ll patients attending a genitourinary medicine (GUM) clinic should be offered an HIV test, according to the National Strategy for Sexual Health and HIV, as part of the initial screening for sexually transmitted infections. ${ }^{1}$ This does not mean that testing is restricted to new patients only and all re-presenting HIV negative patients should be offered and encouraged to have serological testing for HIV and syphilis following possible re-exposure.

Screening of symptomatic and asymptomatic patients attending GUM clinics for HIV is indicated for the following reasons:

- The benefits of early self knowledge of HIV infection in controlling the spread of HIV infection are now recognised. ${ }^{2}$

- There is also enough evidence through cohort studies which show that many people will reduce sexual and needle sharing risk behaviour after a diagnosis of HIV infection $^{3-10}$ and, similarly, those who are unaware of their HIV status do not change their high risk behaviours. ${ }^{611-13}$

- Highly active antiretroviral treatment (HAART) is an important contributor in reducing transmission as a result of the reduction in HIV burden and therefore infectivity in those individuals who are diagnosed early and treated. ${ }^{14}$

- There is also consensus that it is best to start HAART before the onset of severe immunosuppression. ${ }^{15}$

Screening of asymptomatic at-risk groups is most effective if it is coupled with a personalised prevention counselling service. The screening service should provide information regarding the transmission, prevention, and the meaning of HIV test results. ${ }^{16}$ This information should form part of a leaflet that everybody should receive. Additional information should be offered to those refusing a test as lack of perceived risk has been found to be the main reason for test refusal. ${ }^{17}$ Confidentiality of patients must be ensured and informed consent must be obtained beforehand according to the DoH Guidelines for Pretest Discussion. ${ }^{18}$

\section{RECOMMENDED TESTS}

Only Conformité Européenne (CE) marked tests should be used for diagnostic purposes. There are a number of different HIV antibody tests available in the United Kingdom and all have similar sensitivities $(99.78 \%-100 \%)$ and specificities $(99.5 \%-99.93 \%)$ when they are performed according to the manufacturers specifications. ${ }^{19}$ Most laboratories use enzyme immunoassays (EIA) for screening although some of the rapid types of tests are also used for same day test results. A clinical pathology accreditation (CPA) accredited laboratory should perform these tests and the specific test choice will be dictated by local circumstances. The screening assay should be able to detect both anti-HIV-1 and anti-HIV-2 antibodies (third generation test) and preferably p24ag (fourth generation test). ${ }^{20}$ Initial repeated screen positive tests should be referred to a specialist laboratory for confirmatory testing.

\section{Interpreting test results}

When interpreting test results the requesting physician should always remember that no diagnostic test is $100 \%$ accurate, and although the tests have sensitivities and specificities close to $100 \%$, false positive and false negative tests can still occur. In the United Kingdom where HIV prevalence is low a general rule is that low false positive screening tests (negative on confirmatory tests) tend to occur, while false negative tests (unless a person is in the window period) are extremely rare.

\section{Negative HIV test results}

Patients whose specimens test non-reactive (negative) on the initial HIV screening assay should be regarded as noninfected unless the patient presents with symptoms of primary HIV infection (PHI) when it should be repeated after a week (evidence level IV, recommendation grade C).

If a recent exposure to an infected partner or partner of unknown HIV status has occurred within the previous 3 months, the patient may still be in the window period where HIV antibodies have not yet been produced, but p24 antigen (detected as part of the fourth generation or "combo" tests) and/or HIV RNA may test positive. ${ }^{1621}$ Repeat testing after at least 3 months has elapsed since the exposure (see frequency of repeat testing later) should be performed (evidence level IV, recommendation grade $\mathrm{C}$ ).

HIV seroconversion is detected in about $50 \%$ of cases about 1 month after exposure using third generation tests $^{22}$ and 3-4 weeks after exposure using fourth generation tests. $^{23}$

Cases of prolonged or no seroconversion have rarely been reported. ${ }^{24}{ }^{25}$ These initial reports were all tested with older generation antibody tests and many of these long window period cases tested HIV RNA negative on retesting, suggesting infection was caused by a re-exposure at a later date. It is therefore important to stress that the majority of the population will seroconvert within 3 months; however, repeated re-exposure is common and that can seemingly prolong the seroconversion period. In cases where post-exposure prophylaxis (PEP) was given it is still recommended that a 6 month follow up period should be allowed to exclude the majority of seroconversions ${ }^{21}$ simply because of the lack of literature to prove otherwise and because antiretrovirals may reduce replication and prolong antibody response (evidence level IV, recommendation grade $\mathrm{C}$ ).

If a patient presents with clinical symptoms suggestive of HIV infection or AIDS and the HIV screening tests are repeatedly negative, then referral of the specimen to a specialist testing unit is recommended (evidence level IV, recommendation grade $\mathrm{C}$ ).

Abbreviations: CPA, clinical pathology accreditation; EIA, enzyme immunoassays; GUM, genitourinary medicine; HAART, highly active antiretroviral treatment; HIV, human immunodeficiency virus; LIA, line immunoassay; PCR, polymerase chain reaction; PEP, post-exposure prophylaxis; PHI, primary HIV infection; WB, western blot 
Positive HIV test results

The approach in England and Wales is to employ at least two confirmatory HIV antibody tests following the initial reactive screening assay. ${ }^{20}$ The third confirmatory assay may or may not be a highly specific test such as a line immunoassay (LIA). This approach is recommended by the World Health Organization $^{26}$ and the underlying principle has been thoroughly substantiated. ${ }^{27-29}$

It is important that the referral confirmatory laboratory distinguish between HIV-1 and HIV-2 infections. A positive diagnosis of HIV-2 can be made by means of an LIA, western blot (WB), or rapid test devices that incorporate separate type specific reaction spots. ${ }^{20}$ The genitourinary medicine (GUM) clinic should be aware if the referral laboratory is not able to distinguish between HIV-1 and HIV-2 infections, since the viral load assays and treatment need to be tailored for people with HIV-2 infections. Patients who are HIV positive and at risk of HIV-2 infections, such as those from Portugal or west Africa, should have their blood specimens sent to a laboratory that can make the distinction.

A second specimen for confirmation of HIV seropositivity should always be tested to exclude mislabelling and misidentification of the patient ${ }^{20}$ (evidence level IV, recommendation grade $\mathrm{C}$ ).

Indeterminate and unconfirmed HIV test results

The occurrence of false positive or non-specific reactions in the screening assays is not that uncommon, since most of the HIV screening is done in populations with a low prevalence $(<1 \%)$. The usual scenario is that of a low positive signal (repeated twice) in a screening assay while the second and a third assay are negative. At this stage, if primary HIV infection is not suspected, patients should not be told that they are HIV positive, but rather that a false positive reaction is most likely. A repeat blood sample should be sent to the laboratory for exclusion of seroconversion. In the interim period, the patient should refrain from unprotected sex that might put their partners at risk of infection. Most patients who are truly infected with HIV-1 will develop a confirmed HIV antibody positive profile within 1 month. ${ }^{30-32}$ However, evolving signals in the EIAs or evolution to specific HIV antigens in the WB/LIA develop quickly in cases of seroconversion and therefore an anxious patient can be reassured of a non-specific reaction after a repeat sample taken at least 1 week after the first sample if there is nonevolving serology. Once again, it is important to ensure that another follow up blood is tested at least 3 months after the last exposure to exclude infections in the window period (evidence level IV, recommendation grade C).

In cases where an initially weakly reactive test becomes reactive in all of the confirmatory assays seroconversion can be diagnosed. At this stage, it is also common to detect p24 antigen that needs to be neutralised to increase specificity. At this stage, it should be decided whether to enrol the patient into the MRC seroconversion cohort or other available treatment studies.

Nucleic acid testing for HIV-l RNA (viral load assay) or HIV-1 DNA can help to distinguish non-specific reactions from seroconversion. A low level HIV viral load result may well be falsely positive in the situation of possible seroconversion. The caveat is that HIV-1 viral load assays are not validated for HIV diagnosis and it is best performed on a follow up EDTA blood sample.

GUM clinics that make use of same day testing should ensure that the patient is made aware that a delay in providing a test result on the same day does not, by definition, mean that the result is positive and that it happens not uncommonly.
RECOMMENDED SPECIMENS FOR TESTING

Other body fluids, such as urine, oral fluid, and finger stick blood, although routinely used in the other countries including the United States, have mainly been used for seroepidemiological studies in the United Kingdom.

Many local CPA accredited laboratories offer a same day result service and it should rather be used by GUM clinics than performing point of care rapid tests. Rapid point of care tests should only be performed after an appropriate training and safety and quality control programme has been instituted. It would be best practice to involve the local laboratory with setting up and management of such a programme. It should be remembered that the rapid point of care tests do not test for p24 antigen and therefore are not as sensitive as fourth generation EIA tests around the time of PHI.

\section{FACTORS THAT ALTER TESTS RECOMMENDED OR SITES TESTED}

A GUM clinic may not be able to comply with the Department of Health's sexual health directive to test all patients attending the clinic because of a restraint of resources. In these circumstances priority should be given to the following risk groups:

- Patients whose symptoms are compatible with acute retroviral illness or immunosuppression

- Patients who practise unsafe sex-that is, unprotected anal/vaginal sex with multiple partners, past/current history of STD, sexual assault

- Patients who are known contacts of HIV infected patients

- Injecting drug users who share "equipment"

- Patients who come from countries with a high HIV prevalence

- Patients who travel abroad with exposure to high risk activity.

\section{RECOMMENDATION FOR FREQUENCY OF REPEAT TESTING IN AN ASYMPTOMATIC PATIENT}

(EVIDENCE LEVEL IV, RECOMMENDATION GRADE C)

A positive test should be followed up by a repeat HIV test to exclude the possibility of a specimen mix up.

A negative test cannot exclude a recent infection if the exposure was less than 3 months ago (see interpretation of tests).

The timing and frequency of retesting has not yet been firmly established. ${ }^{16}$

The following factors should be taken into consideration when recommending follow up testing:

- Timing of last potential exposure. If it is thought that a recent possible exposure has happened, then a patient with a negative test should undergo a repeat test in at least 3 months' time.

- Probability of HIV infection given type of exposure. Patients who have had a definite HIV exposure and in those cases where PEP was given, need to be followed up at 3 and 6 months. ${ }^{33}$

- Ongoing high risk behaviour. One of the aims of counselling is to modify high risk behaviour, but if there is continuation then frequent testing would be advocated.

- Patients who are very anxious might be retested sooner following a indeterminate test result (that is, after l week)—see under indeterminate results.

- When a patient presents again to a GUM clinic then by definition they should be treated as a new patient and be retested for HIV. 


\section{RECOMMENDATION FOR TEST OF CURE}

There is no test of cure, but all HIV antibody positive patients should be referred on to a specialist HIV treatment and care centre for further HIV-1 viral load testing and management. It is important to make sure that the referral laboratory stores all HIV viral load plasma indefinitely for future retrospective resistance testing should the need arise.

\section{STAKEHOLDER INVOLVEMENT}

No stakeholders were involved in the drawing up of these guidelines.

\section{RIGOUR OF DEVELOPMENT}

The guidelines are based on all available scientific sources and where evidence is lacking, opinion of "best practices" by specialists in the field was used. Two main documents were consulted, CDC's Revised guidelines for HIV counselling, testing (Nov 2001) and Towards error free HIV diagnosis: guidelines on laboratory practice, produced by the HPA HIV Laboratory Diagnostic Forum. Publications from the CDC, HPA, and DoH were searched by means of their respective internet search engines for keywords "HIV +/- guideline +/testing". Likewise a Medline search was undertaken (November 2003) with the search criteria: "HIV + testing + guidelines" and the titles of the first 200 "hits" were reviewed, of which 27 articles were selected for abstract review.

Special mention on the 3 month follow up PEP should be made. The CDC's guidelines states that following a sexual exposure a 6 month follow up period should be allowed to exclude HIV infection. The HPA guidelines state that at least 6 months needs to pass following a needlestick injury to exclude infection, a period also accepted in these guidelines. However, following sexual exposure, the HPA guidelines are not clear whether the recommendation of "testing immediately after the exposure and then: at 1-2 months, at 34 months, and 6 months" only pertains to needlestick injuries or also to sexual exposures.

As mentioned in these guidelines, the 6 month waiting period is based on the initial studies of Busch (1995), Simmonds (1988), and Horsburgh (1989), which used "known" exposure dates to calculate seroconversion periods. Of the three studies, Busch seems to be the most reliable and from a subsequent review of their and other data a conclusion was drawn that states that seroconversion in a third generation assay would (in about $50 \%$ of cases) occur 1 month after exposure and 4-8 days earlier using a fourth generation assay. The drawback of the other studies was that they were performed when less sensitive (first and second generation) tests were used, it was not taken into account that most people will only seroconvert following repeated sexual exposures, and retesting initial polymerase chain reaction (PCR) test positive samples did not confirm the results. This can be explained by the fact that initial PCR reactions were crude and gave many false positive reactions, which meant that the infected patients most probably became infected at a much later stage when they were reexposed to HIV.

At the Birmingham HPA laboratory, we have employed an "at least" 3 month follow up period after the last sexual exposure for a few years and we have not had any known patients seroconverting beyond this time. Dr Philip Mortimer (ex-director Sexually Transmitted and Blood Borne Virus Laboratory, HPA) is also not aware of any seroconversion beyond 3 month exposure cases and he is of the opinion that the 3 month follow up period is perfectly reasonable following a sexual contact (personal communication).

Selecting the phrase "at least" 3 month follow up also does not go against the DoH guidelines for pretest discussion, ${ }^{18}$ which states: "If thought [to be] a recent possible exposure, a patient could be in the window period [and] they should be advised to undergo a repeat test in three to six months' time."

\section{APPLICABILITY \\ Auditable outcome measures}

- All HIV positive laboratory diagnoses should be recorded and patients contact traced.

- Each new patient seen should be offered an HIV antibody test with appropriate pretest discussion, unless they have already been diagnosed as being infected with HIV.

- At least $60 \%$ of all patients who tested HIV negative following a high risk exposure, but where at least 3 months since the exposure has not yet passed since they were last tested, should be retested.

Conflict of interest: none.

Correspondence to: E J Smit, HPA Heartlands Hospital, Bordesley Green East, Birmingham B9 5SS, UK; erasmus.smił@heartofengland. nhs.uk

Accepted for publication 31 August 2006

\section{REFERENCES}

1 Public Health Laboratory Service. The national strategy for sexual health and HIV. Sexual health and HIV Strategy Integrated Steering Group United Kingdom. London: PHLS, 2001.

2 Valdiserri RO, Holtgrave DR, West GR. Promoting early HIV diagnosis and entry into care. AIDS 1999; 13:2317-30.

3 Rietmeijer CA, Kane MS, Simons PZ, et al. Increasing the use of bleach and condoms among injecting drug users in Denver: outcomes of a targeted, community-level HIV prevention program. AIDS 1996;10:291-8.

4 Rhodes F, Malotte CK. HIV risk interventions for active drug users. In: Oskamp S, Thompson S, eds. Understanding HIV risk behavior: safer sex and drug use. Thousand Oaks, CA: Sage Publications, 1996:297-36.

5 Gibson DR, Lovelle-Drache J, Young M, et al. Effectiveness of brief counseling in reducing HIV risk behavior in injecting drug users: final results of randomized trials of counseling with and without HIV testing. AIDS Behav 1999:3:3-12.

6 Doll LS, O'Malley PM, Pershing AL, et al. High-risk sexual behavior and knowledge of HIV antibody status in the San Francisco City Clinic Cohort. Health Psychol 1990;9:253-65.

7 Cleary PD, Van Devanter N, Rogers TF, et al. Behavior changes after notification of HIV infection. Am J Pub Health 1991:81:1586-90.

8 Fox R, Odaka NJ, Brookmeyer R, et al. Effect of HIV antibody disclosure on subsequent sexual activity in homosexual men. AIDS 1987;1:241-6.

9 Van Griensven GJP, de Vroome EMM, Tielman RAP, et al. Effect of human immunodeficiency virus (HIV) antibody knowledge on high-risk sexual behavior with steady and nonsteady sexual partners among homosexual men. Am J Epidemiol 1989;129:596-603

10 Coates TJ, Morin SF, McKusick L. Behavioral consequences of AIDS antibody testing among gay men [Letter]. JAMA 1987;258:1889.

11 Wenger NS, Kusseling FS, Beck K, et al. Sexual behavior of individuals infected with the human immunodeficiency virus: the need for intervention. Arch Intern Med 1994;154:1849-54.

12 Desenclos J-C, Papaevangelou G, Ancelle-Park R, for the European Community Study Group on HIV in Injecting Drug Users. Knowledge of HIV serostatus and preventive behaviour among European injecting drug users. AIDS 1993;7:1371-7.

13 Dawson J, Fitzpatrick R, McLean J, et al. The HIV test and sexual behavior in a sample of homosexually active men. Soc Sci Med 1991;32:683-8.

14 Quinn TC, Wawer MJ, Sewankambo N, et al. Viral load and heterosexual transmission of human immunodeficiency virus type 1. N Engl J Med 2000;342:921-9.

15 BHIVA Writing Committee. British HIV Association (BHIVA) guidelines for the treatment of HIV-infected adults with antiretroviral therapy, July 2003.www.bhiva.org/guidelines/2003/hiv/index.html.

16 Center for Disease Control. Revised guidelines for HIV counseling, testing and referral. MMWR 2001;50(RR19):1-58

17 Wickramasinghe TK, Rogstad KE. Which patients attending genitourinary medicine clinics have HIV tests? Int J STD AIDS 2002;13:843-6.

18 Department of Health. Guidelines for pretest discussion on HIV testing. London: DoH, March, 1996.

19 Perry KR, Kenny C, Parry JV, et al. bioMerieux VIDAS HIV DUO. Medical Devices Agency evaluation report 1999;MDA/99/69.

20 Parry JV, Mortimer PP, Perry KR, et al. Towards error free HIV diagnosis: guidelines on laboratory practice. Commun Dis Public Health 2003;6:334-50. 
21 Lindback S, Thorstensson R, Karlsson AC, et al. Diagnosis of primary HIV-1 infection and duration of follow-up after HIV exposure. Karolinska Institute Primary HIV Infection Study Group. AIDS 2000;14:2333-9.

22 Busch MP, Lee LL, Statten GA, et al. Time course of detection of viral and serological markers preceding human immunodeficiency virus type 1 seroconversion: implications for screening of blood and tissue donors. Transfusion 1995;35:91-7.

23 Busch MP, Statten GA. Time course of viremia and antibody seroconversion following human immunodeficiency virus exposure. Am J Med 1997:102:117-24.

24 Horsburgh CR Jr, Jason J, Longini I, et al. Duration of human immunodeficiency virus infection before detection of antibody. Lancet 1989;2:637-40.

25 Reimer L, Mottice S, Schable C, et al. Absence of detectable antibody in a patient infected with human immunodeficiency virus. Clin Infect Dis 1997; 25:98-100

26 Joint United Nations Programme on HIV/AIDS (UNAIDS) - WHO. Revised recommendations for the selection and use of HIV antibody tests. Weekly Epidemiol Record 1997;73:81-8.
27 Groen G van der, Kerckhoven I van, Vercauteren G, et al. Simplified and less expensive confirmatory testing. Bull World Health Organ 1992;69:747-52.

28 Nkengasong J, Kerckhoven I van, Vercauteren G, et al. Alternative confirmatory strategies for anti-HIV antibody detection. J Virol Meth 1992;36:159-70

29 Urassa WK, Bredberg-Raden U, Mbena Aphraim, et al. Alternative confirmatory strategies in HIV-1 antibody testing. J AIDS 1992;5:170-6.

30 Celum CL, Coombs RW, Lafferty W, et al. Indeterminate human immunodeficiency virus type 1 western blots: seroconversion risk, specificity of supplemental tests, and an algorithm for evaluation. $J$ Infect Dis 1991;164:656-64.

31 Jackson JB, MacDonald KL, Cadwell J, et al. Absence of HIV infection in blood donors with indeterminate western blot tests for antibody to HIV-1. N Engl J Med 1990:322:217-22.

32 Dock NL, Kleinman SH, Rayfield MA, et al. Human immunodeficiency virus infection and indeterminate western blot patterns: prospective studies in a low prevalence population. Arch Intern Med 1991;151:525-30.

33 UK Health Departments. HIV post exposure prophylaxis-guidance from the UK Chief Medical Officers' Expert Advisory Group on AIDS, July, 2000. 